\title{
The Design of the Infusion Alarm
}

\author{
Xuetao Liu \\ North China Electric Power University, Beijing, 102206 \\ alanncepu@foxmail.com
}

Keyword: Infusion alarm; Optocoupler; Microcontrollers; Wireless transmission

\begin{abstract}
In order to ensure the safety of patients and make the patients have a good rest during liquid infusion, we design a wireless infusion alarm system. The system adopts two terminal mode with host and slaves. The slaves in the patients' side are in charge of detecting whether the infusion is completed, while the host in the nurse side are in charge of monitor the slaves. The system use optocoupler MOCH22A to detect the liquid in the infusion hose. Through the combination of analog circuit and digital circuit, the information is transmitted to the microcontroller. Bluetooth is connected with microcontrollers so that the host and slaves can be connected by wireless. When the infusion is finishing, the corresponding slave and the host simultaneously alarm and notify the nurse to deal with it in time.
\end{abstract}

\section{Introduction}

Venous transfusion is widely used in the clinical nursing work, the most common means of medical treatment, but at present, most hospitals and medical institutions don't realize automatic monitoring management of transfusion .So after established venous channel for patients, patients and their families must pay attention to transfusion progress all the time in case the medicine liquid used up then leading to danger, which is bad for patient to recuperate. What's more, nursing staff only rely on experience or frequent wards to grasp the transfusion progress of patients, which means a lot of invalid workload for nurses. And if the nurse failed to find out the end of patients' infusion timely, there will be a lot of dangerous problems because once the infusion problems occurred, air may go into the blood vessels which results in air embolism and needles blocked by clotting. If that happened, it will delay treatment, break out the contradiction between nurses and patients, lead to medical accidents, even endanger patients' lives. So our work strive to reduce the burden of patients and nurses, alarm intelligently by a form of automation. It can inform nurses it's time to pull out the needle or change medicine timely by detecting the level of medicine liquid .

\section{Design and Achievement of System}

The Whole Design of System. Overall, this design is divided into two parts, multiple slaves in the patients' side to detect and a host in the nurses' side to monitor. Slaves are consisted of opticalcoupler circuit, 51 microcontrollers module and Bluetooth, while the host are consist of 51 microcontrollers module and Bluetooth. When opticalcoupler detected liquid is dropping out, the bell in the circuit alarms and the circuit transfer the information to the microcontrollers in slave side. The microcontrollers will drive the Bluetooth module so that the information can transfer from the slave to the host by Bluetooth. After the microcontrollers get the information from the Bluetooth in host side, it will alarm too. The process of information transfer in the system is shown in the Fig. 1 below. 


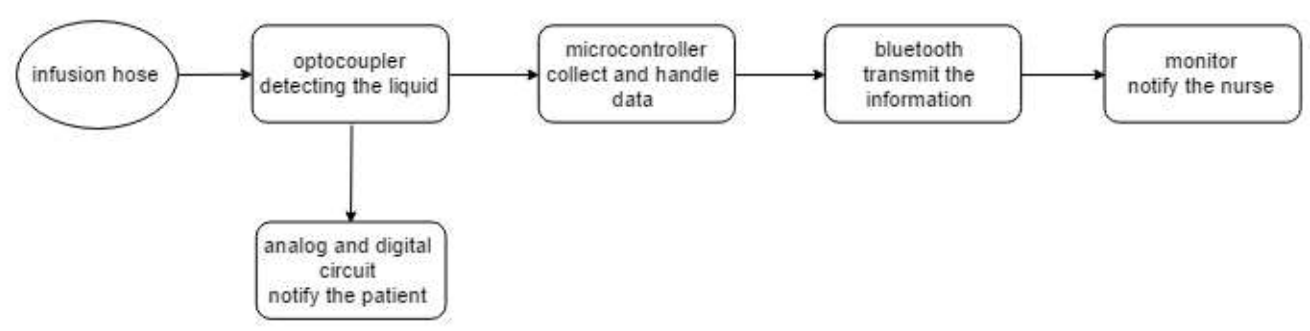

Figure 1. The process of information transferability

From a technical level, the design is divided into optical coupler circuit to detect liquid level and microcontrollers to transfer the information, so this article will mainly analysis from the two aspects.

The Design of Detecting Circuit. The core of the detecting part is optical coupler MOCH22A. MOCH22A is a kind of photoelectric coupling device, as shown in Fig.2, the right part of it is an infrared light generator, the left is photosensitive transistor. Infusion hose is in the middle of the MOCH22A. Whether the medicine liquid exist makes the refraction of the infrared ray different, and then the intensity of the light on the photosensitive transistor is different too, which affects the resistance on the photosensitive transistor, we find a appropriate position and measure the resistance of photosensitive transistor in different situations. The result is shown below in Table.1.

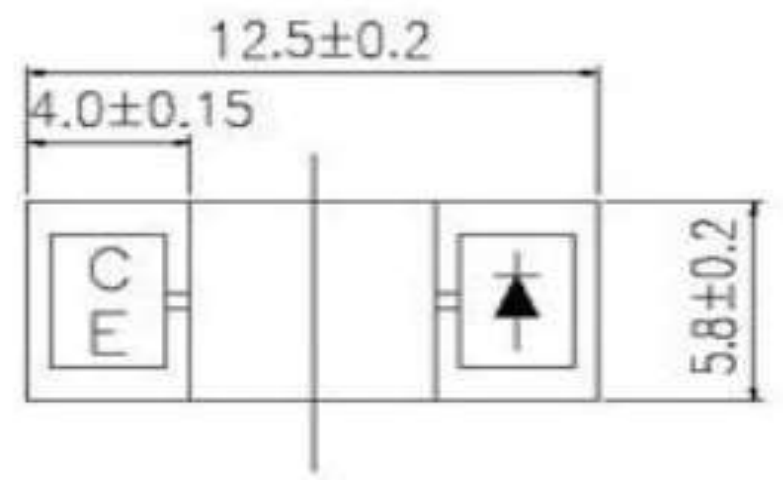

Figure 2. The structure of $\mathrm{MOCH} 22 \mathrm{~A}$

Table 1 The resistance of transistor

\begin{tabular}{|c|c|c|}
\hline Whether liquid exist & Yes & No \\
\hline The resistance of transistor & $11 \mathrm{k} \Omega$ & $20 \mathrm{k} \Omega$ \\
\hline
\end{tabular}

We design a circuit including MOCH22A according to the parameter above. The circuit is shown below.

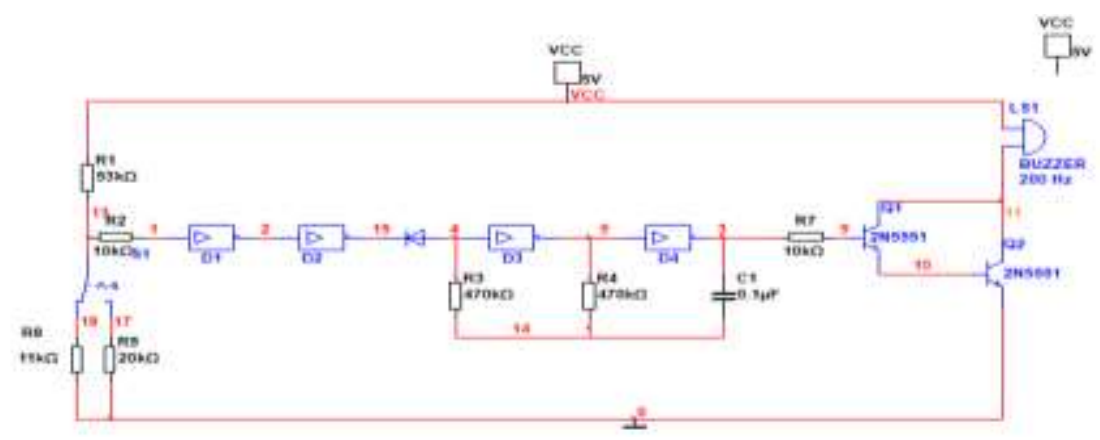

Figure 3. The detecting circuit of slaves 
Because there is no MOCH22A in simulation software Multisim, so with two resistance and a SPDT to replace, Multisim simulation result shows that when the SPDT connect to resistance of 11 $\mathrm{k} \Omega$, the buzz don't alarm, while it's opposite when connecting to the resistance of $20 \mathrm{k} \Omega$. So that's prove the design is effective. The detailed instruction of the circuit is shown below.

The detecting part of the system was designed by a refractive principle. MOCH22A, groove shape photoelectric coupler, is used to realize the detecting function. The infusion tube is put in the groove. Make the photosensitive triode get the strongest light by adjusting the position of infusion tube when the infusion hose is full of liquid. At this time the photosensitive triode shows low resistance. Its equivalent resistance share the voltage with the resistance R2 so that the inverter D1 can input a low level and output a high level while inverter D2 input a high level and output a low level. The diode is forward biased and limit the oscillating circuit to oscillate. So, D3 input low level while D4 output low level. And triode emitter reverse bias. The bell does not sound.

Next the situation that there is no liquid in infusion hose will be introduced.

Because the refractive index of air is less than the liquid, the original index system is different from before. The infrared light that photosensitive transistor get is weak so that equivalent resistance of photosensitive transistor is high and voltage that it shares with resistance R2 is high, too. The inverter D1 input high level while D2 output high level. The diode reverse bias eliminates the control of the oscillator. The oscillator begin to oscillate and output a signal to Darlington tube which is made up of VT1 and VT2 to enlarge the signal. Then the buzz that connect with Darlington tube and source will alarm. The output of the circuit is connected with the microcontroller, and the microcontrollers detect the output to collect the situation of the medicine liquid in infusion hose, and then transfer the information.

Data Transmission. The design uses HC-05 Bluetooth integrated module to realize wireless transmission. Through the instruction set of AT, the transmission rate, transmission word length, check mode and the other parameter which match with the Microcontrollers are set up. The connection between Microcontrollers and Bluetooth are common, limited to space, we don't introduce too much. Next, the data design and transmission of Microcontrollers will be emphatically introduced.

\section{Communication Mode}

In this design, the host and slave use polling mode to communicate. That means the host sends the communication request to the specific slave automatically, T0 timers are in charge of timing, and polling every 5 seconds. 51 single chip Microcontrollers adopts serial communication mode three to communicate. Serial communication mode three is the asynchronous communication mode of 11-bit data. In the 11-bit data, there is 1 start bit, 9 data bits (including 1 bit additional ninth bit, which is TB8 when transmitting while RB8 when receiving in SCON.) In communication, SM2 is used to communicate with specific slave in SCON (Serial Control Register). When the slave is in a wait state, SM2 sets 1 . When the host try to communicate with the slave, it sends the slave's address, and sets the TB8 at 1. In this way, the slave will send the received address data to SBUF and compare it with its address. If it is not its own address, it continues to wait, otherwise it send a successful transmission flag (0x0f), set SM2 at 0, and then start the data transmission with the host. When the host transfers data, the TB8 is assigned 0. So, the data sent is accepted by the slave only.

Data Design. When designing data transmitted from a slave computer, the transmitted data needs to include the status of the slave and the information of the floor and room of the slave. At the same time, in order to improve the flexibility of the application, the design needs to guarantee that the floor and room numbers can be changed. Therefore, the first bit of the data of the 8 binary bits (the first bit is the highest bit, the second bit is the secondary high, and so on) is set as the state bit that is used to indicate whether the slave is in the alarm state. If the slave is in alarm state, the highest bit is set as 1 , otherwise it will be set as 0 . The fourth place is the floor number $(0$ corresponds to the first floor, while 1 corresponds to the 2 floor). The last three bits are set to room number (room number can be 1 to 5 ). So, there are ten rooms in total. The others are arranged to reserve. It can be extended when the number of rooms or floors are increased. Before the single 
chip microcontrollers works, it must set the floor and room number first. In this design, set three buttons P3.0, P3.1, p3.2 to achieve these functions. Once P3.0, enter the floor number setting status. After once P3.1, the floor number increases 1. After once P3.2, the floor number decreases 1. After the second P3.0, enter the room number settings, the room number can change by button P3.1 and P3.2. After third times of P3.0, the slave initialization is completed, and the state of waiting for the host's call is officially entered.

When designing the data sent by the host, both the slaves' state and address should be included in the data. The highest bit of the data in the 8 binary bits is used to be the state bits of the address (when the highest bit is 1, it means the address is idle. When the highest bit is 0 , it means that the address has been allocated.), while the remaining 7 bits are used as the slave address. When a slave dropped, the highest bit of the slave's address data in address array is set to be 1 . The data will be placed at the last of the address array, the address data behind that will be moved forward one by one, and the total number of slaves will be reduced by 1 . When there is a new slave go online, the total number of slaves will be increased by 1 . The highest bit of Nth address in the address array is assigned 0 , and the address is assigned to the slave.

The Design of the Display. In order to make the slave display the state and the host display the received data, the LCD screen is added into the design. A 1602 LCD with two rows of data can be used on the slaves. The first row displays the address, the floor number and the room number, and the second line displays the slave state. The host use a 12864 LCD which can display 4 rows of data,

The first row is room number, the second row is the state of slaves' in first floor, the third row is the state of slaves' in second floor ('-' stands for 'not line', '0' stands for 'no alarm', ' 1 ' stands for 'alarm'), and the fourth row display the slaves go online or go offline. In order to display conveniently, the host designed a buffer array to sort the slave according to the floor and room number.

In order to display timely, after the end of each polling and the slaves' communication with the host, the state of the display screen should be updated.

The Host's Response. After a slave alarm, the state bit which is in both the slave and host changes from 0 to 1 . But it must reset 0 so that the system can alarm again. So host should response it. When the host is interrupted, the slaves' response can be completed by the button P3.0, P3.1. When the P3.0 is pressed once, the first slave that is in alarm state will be found. And then press the P3.1, the state bit of the slave in host will be set as 0. Meanwhile, host will communicate with the slaves and set the state bit of the slave in slave as 0 , too. The next slave whose state bit is ' 1 ' will be found by pressing P3.0 again. Press P3.0 when the last salve whose state bit is ' 1 ', it will return the first slave whose state bit is ' 1 '. After pressing P3.1 twice, the interrupt will be out. After above, the response of host is completed.

\section{Conclusion}

Our design has realized the function of infusion alarm. Through the wireless transmission of Bluetooth, it can alarm at both patients' side and nurses' side, which can reduce the workload of nurses, and make patients get a better rest. The product has sensitivity, reliability and convenience by using Bluetooth and optocoupler. At present, there is no infusion alarm with wireless function in the market, so if it can be mass produced, it can quickly make up for the blank of the market and have a good market potential.

\section{Reference}

[1] ttps://wenku.baidu.com/view/bff4cc3f5727a5e9856a61bc.html

[2] ianxiang Guo. New concept 51 singlechip C language tutorial[M] Publishing House of Electronics Industry 2009 
[3] iali Tang, Houchuan Yang. 51 microcontroller C language easy access [M]. Publishing House of Electronics Industry, 2016.

[4] hibai Tong, Chengying Hua. Fundamentals of Analog Electronic Technology (Fifth Edition) [M] Higher Education Press,2013

[5] ueqiao Li. Fundamentals of digital electronic technology (Second Edition) [M] China Electric Power Press, 2013

[6] o Yang, Yanan Sun, Pinchao zeng,etc. Development of a new infusion alarm system [J]. China Medical Equipment, 2009, 6(2):9-11.

[7] zhou Cui. Basic course of $\mathrm{C}$ programming language design [M] Higher Education Press. 2007

[8] ong Jiang. Research on transfusion alarm monitoring system based on photoelectric sensor [J]. Chinese Journal of Medical Device, 2010, 23(12):9-11.

[9] i Deng. Protel 99 SE schematic diagram and PCBDesign and simulation [M]. Posts \& Telecom Press, 2013.

[10] ttps://wenku.baidu.com/view/ecbeda6a58fafab069dc02a1.html 